\title{
Like It or Not: Issue of Credibility in Facebook Advertising
}

\author{
Azizul Yaakop ${ }^{1}$, Marhana Mohamed Anuar ${ }^{1} \&$ Khatijah Omar $^{1}$ \\ ${ }^{1}$ Universiti Malaysia Terengganu, Malaysia \\ Correspondence: Azizul Yaakop, Universiti Malaysia Terengganu, Malaysia. E-mail: azizul_yadi@umt.edu.my
}

\author{
Received: December 31, 2012 Accepted: January 28, 2013 Online Published: February 28, 2013 \\ doi:10.5539/ass.v9n3p154 \\ URL: http://dx.doi.org/10.5539/ass.v9n3p154
}

\begin{abstract}
Issues like advertising credibility and privacy trust have become the hot topics for social networking sites (SNSs) of late. In spite of the critiques, the trend of employing SNS's as advertising platform by marketing practitioners is still on the rise. This approach of adopting SNS's probably suits marketers' objectives to reach and communicate their users and potential customers with relevant ads and personalized messages. Besides, this approach is expected to increase the value of advertising for both users and marketers in terms of profit and return on investment. However, research studies on SNS's and how they are perceived by its users are relatively limited, especially how online factors influence users' perceptions and attitudes towards advertising on SNS's. This paper aims to examine the online factors that influence consumers' perceptions and attitudes towards advertising on Facebook. A total of 350 respondents participated in the study. The results suggest that there are three online factors that significantly influence consumers' attitudes towards advertising on Facebook. The factors are perceived interactivity, advertising avoidance and privacy. Surprisingly, credibility was not a significant factor predicting consumer' attitudes towards advertising on Facebook. This paper provides some insights to advertisers into dimensions that may draw consumers' favourable attitudes towards advertising on SNS's, especially Facebook.
\end{abstract}

Keywords: attitudes towards advertising, social networking sites (SNS), Facebook, advertising credibility

\section{Introduction}

With the rapid development of information technologies worldwide in the past decade, advertisers are increasingly relying on various modes of interactive technology to advertise and promote their products and services. Furthermore, the idea of executing a content that is current and entertaining could get consumers to interact electronically in an effective way. This powerful attribute can be seen as a future of advertising and may become more figurative in consumers' minds than television advertising as a marketing stimulus that stands out relative to others in their environment (Yaakop \& Hemsley-Brown, 2011).

Issues like advertising credibility, privacy trust, advertising avoidance and interactivity have been the highlights in most of previous research on online advertising. These issues are imperative because they may act as barriers or boosts in delivering effective messages to audiences and in building awareness on the company's offerings. By means of social network sites such as Facebook and Twitter, marketing practitioners have experienced a leap in their engagement with advertising activities. For example, click-through rate (CTR) for advertising on Facebook is now an alternative to banner ads which has fallen for the past over time. However, research studies on these social network sites and how it is perceived by its users is relatively limited and unavailable. This statement is supported by Boyd and Ellison (2007) that stated that there is little research published in the area of consumer perceptions of advertising on social networking sites although these sites faces rapid growth over a very short period of time. Besides that, although research exists in the area of online communications, not all of this work is easily transferable to the social media space (Bond, Ferraro, Luxton \& Sands, 2010). Also, little is known about how online factors influence Internet users' attitudes toward online advertising (Campbell \& Wright, 2008). Besides that, capitals investment in online advertising are often targeted wrongly due to lack of knowledge and limited research done on consumers' opinions of online advertising which caused advertisers chosen the wrong advertisement characteristics; sending unclear messages; and cannot clearly identify the target audience (Hadija, 2008). Hence, the aim of this study is to fulfill the research gap by investigate the relationship between user perception and their attitude on online social networks advertising using the context of Facebook. 


\section{Literature Review}

A previous study by Zeng et. al (2009) found that responses to online advertising via social network heavily depended on perceptions of advertising relevance and value, as well as being influenced by social identity and group norms. Unlike, Hadija (2008) found perceived interactivity as an important influence in consumers' responses to the same subject. Other dimensions such as advertising avoidance, credibility and privacy were used in the past to measure consumers' perceptions towards online advertising (Kelly, 2008; Roberts, 2010).

Therefore, in congruence with the previous literature, this research is proposing that consumers' attitudes towards advertising on Facebook is presented as a two-level structure depicting generalized perception of advertising on Facebook and the individual's perceptions with advertising .

\subsection{Perceived Interactivity}

On a personal level, interactivity is described as means for individuals to effectively communicate with each other, regardless of distance or time (Ha \& James 1998). On a mechanical level, interactivity is described as a characteristic of a medium which allows for its users to participate in creation and recreation of the content (Steuer, 1992). Interactivity on the Internet shifts the ways in which users perceive advertising Hadija (2008). Since offline advertising such as television and radio are different than online advertising, advertisers need to analyze factors that influence consumer acceptance towards an online advertising, which would include social media like Facebook. According to Mangold and Faulds (2009), social media have been acclaimed for having influences on every stage of the consumer decision-making process as well as influencing general opinions and attitude formation. It is believed that high level of perceived interactivity will incur positive and favorable attitudes towards the advertisement on social networking sites (SNSs).

One of the interactive capacities of Facebook is its ability to attract users to use text, images, videos and links as interactive content as strategies to track and share new products with consumers. On Facebook, creating social media profiles and updating them frequently helps improve the general awareness and visibility of the online business and brand. When customers are searching for information about a company, the multilingual social media profiles will provide information about your business, links to your website and different information channels that build credibility. Like other social media websites, Facebook was created primarily to connect people. Therefore by creating personal profiles, it allow marketers to create company pages that can provide direct information about the company, products and services and have links to the website, building a social media campaign for brand awareness.

Facebook has created a way for companies to market and sell their products and services in a new different way. Advertisements that appear on every page of Facebook have enabled companies to reach potential consumers in a way that is less time consuming and cost effective. Because of that, companies can target users that are the most likely to purchase their products by accessing the cookies from the Facebook users web browser. For example, if a Facebook user is checking the same retailer's website a couple times a week, their ads start to appear on the Facebook account. Even more amazing, similar if not the same, products that the user looks up on that website is advertised on Facebook specifically for the user.

\subsection{Advertising Avoidance}

Advertising avoidance includes "all actions by media users that differentially reduce their exposure to advertisement content" (Speck \& Elliott 1997, p. 61). Increasing clutter and media fragmentation now expose consumers to thousands of commercial messages every day (Gritten 2007). A person has the ability to avoid an advertisement by cognitive, behavioral and mechanical means (Speck \& Elliott, 1997). For example, ignoring the advertisement on newspaper or magazine advertising is considered a cognitive method of avoidance. Switching to other television programs during the advertising break is considered a behavioral method of avoidance. And for the internet medium, deleting pop ups on the internet is considered a mechanical method of avoidance.

Cho and Cheon (2004) mentioned that interruption of task, perceived clutter on internet sites and negative past experiences with internet advertising are all antecedents explaining avoidance of advertising on the internet. Factors such as interruption of task happen when the speed of data retrieval and processing in internet is reduced or interrupted by advertising that it requires an action from the consumer before they are able to resume their online activity. Advertising clutter is the term given to the perception of the consumer when they believe that there is too much advertising within a given media at a given time (Cho \& Cheon, 2004). Negative past experiences happens when consumers encounter unfavorable situation such as receiving deceiving messages or exaggerated or been forwarded to inappropriate sites. 


\subsection{Credibility}

Credibility refers to the objective and subjective components of the believability of a source or message. Media and advertising credibility can be defined as the extent to which the consumer believes or trusts in the media or advertising claims (Moore \& Rodgers, 2005). Findings by Johnson and Kaye (2002) suggested that reliance on traditional sources; political trust and convenience were the best predictors of online media credibility. Johnson and Kaye's earlier study on media credibility (1998) found that online sources were considered credible and that younger people are more likely to view online information as credible. The study acknowledged that even though the internet has an unregulated flow of information and that the quality of information was not subject to the same scrutiny shown to traditional media; it still was considered a more credible source of news information.

Even though online news is viewed as being credible (Johnson \& Kaye, 1998), the internet was found to be the least credible medium to advertise in, with consumers regarding it with the highest level of skepticism. Moore and Rodgers (2005) found that the consumers did not feel comfortable about surfing the online advertisements and although as college students they fell into the demographic that shops online the most, they still did not find the internet advertising trustworthy. They were hesitant when required to give credit card details or personal information and only purchased from sites that they knew and trusted (Moore \& Rodgers, 2005).

\subsection{Privacy}

The online privacy debate has existed since the creation of the Internet (Roberts, 2010). In social networking sites (SNSs), privacy concerns has also been an issue since users shares their information and preferences such as their personal details, images, statuses, hobbies and so on. Barnes (2006) stated that youth culture has embraced online social networking and they are now publicly sharing very personal information on these sites. On Facebook, however, it offers an access to user information and generates profile privacy settings in order to overcome the concern of privacy (Ragan 2009). While privacy policies are often criticized as difficult or time consuming to read (Bonneau and Preibusch, 2009; McDonald and Cranor, 2009; McDonald, Reeder, Kelley, and Cranor, 2009), there is evidence that if a website has a privacy policy, individuals are more likely to share personal information with the website (Cranor et al., 2000).

\subsection{Conceptual Framework}

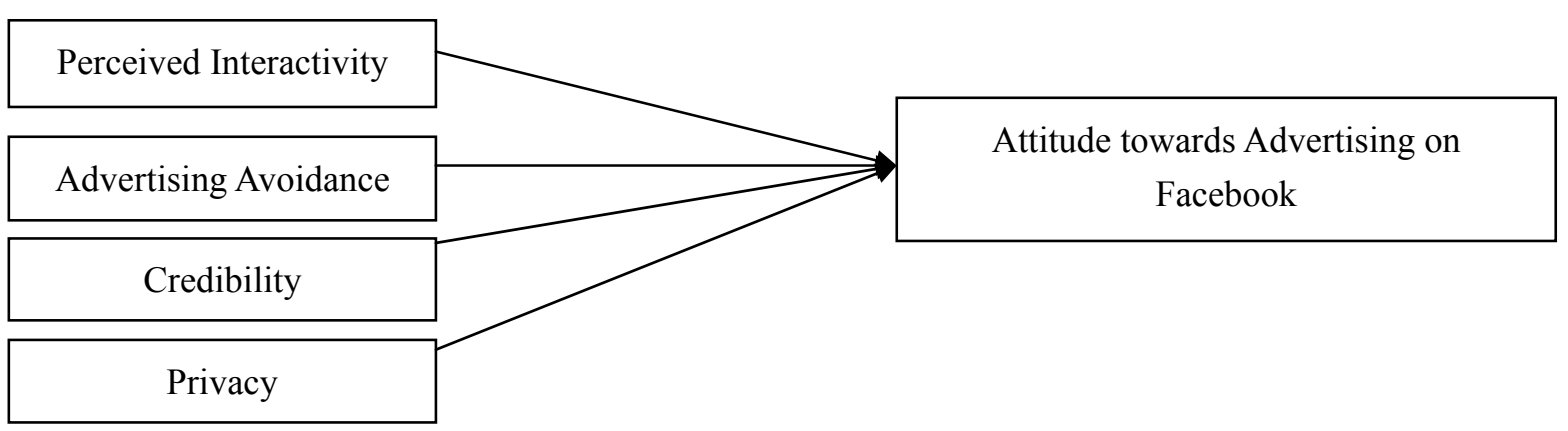

Figure 1. A conceptual framework of attitude towards advertising on Facebook

From the Figure 1 above, this conceptual framework is incorporating perceived interactivity, advertising avoidance, credibility and privacy as the observed factors for attitude towards advertising on Facebook.

A hypothesis is a tentative explanation that accounts for asset of facts and can be tested by further investigation. Selamat (2008) stated that hypothesis is tentative, intelligent guesses posited for the purpose of directing one's thinking and action towards the solution of a problem. In this study, the hypotheses are as follow:

H1: Perceived interactivity significantly predicts attitudes towards the advertisement on Facebook.

H2: Advertising avoidance significantly predicts attitudes towards the advertisement on Facebook.

H3: Credibility significantly predicts attitudes towards the advertisement on Facebook.

H4: Privacy significantly predicts attitudes towards the advertisement on Facebook.

\section{Research Methodology}

Following previous literature on examining attitudes towards advertising, students especially advertising or marketing students were used as the sample group mainly because they represent a major market segment, besides frequently featuring in convenience sampling (Yang 2000). In addition, advertising and marketing 
students were among the groups commonly used to investigate attitudes to advertising because they represent future advertising professionals; thus, measuring their attitudes to advertising would provide for a good start in this research area (Andrews et al., 1991). Besides that, university students are heavy users of communication technologies and undergraduate students are heavy users of the online social network site (Smith, Caruso \& Kim, 2010) which means they can understand the content in Facebook well and its functions. According to Lenhart, Purcell, Smith and Zickuhr (2010), the use of online social networks among teens and young adults has exploded during the last few years and a survey estimated that in the US 72\% people of the 18-29 years old are using online social networks. Calisir (2003) also claimed that students are most frequent users and they represent the biggest segment in the Web usage.

In this research, the sample of the study is undergraduate students who are currently studying the programme of Bachelor in Management Marketing at Universiti Malaysia Terengganu (UMT). By referring to the table by Sekaran (2000), the total number of samples that should be selected is 357 since the total numbers of undergraduates in Universiti Malaysia Terengganu (UMT) is about 5000 undergraduates taking account all faculties in the university. A set of questionnaire is prepared in English and the data collected was analyzed using SPSS 17.0. The questionnaire used in this research was divided into 2 sections and the data was collected by means of a structured questionnaire. The questionnaire contains 24 items in section A and 3 items in section B. Section A is about the perceptions towards advertising on Facebook while section B is about the attitude towards advertising on Facebook. The questionnaire for this study is adopted based on studies from Hadija (2008) and Kelly (2008) and are modified to the topic of this study. Gorard (2001) assured that the advantages of using such previous instruments and questions are considerable because the instruments used have been piloted, used previously, are mature and ready to be used, probably on a far larger scale than the researcher could envisage.

\section{Findings and Discussion}

Questionnaires were distributed to a total of 350 respondents that are currently studying the programme of Bachelor of Management Marketing in the Faculty of Management \& Economics in Universiti Malaysia Terengganu (UMT). The respondents' demographic characteristics are presented in Table 1.

Table 1. Respondents' demographic characteristics

\begin{tabular}{cccc}
\hline \multirow{2}{*}{ DEMOGRAPHICS } & & FREQUENCIES & PERCENTAGE (\%) \\
\hline \multirow{2}{*}{ Gender } & Male & 229 & 65.4 \\
& Female & 121 & 34.6 \\
& Malay & 91 & 26.0 \\
& Chinese & 189 & 54.0 \\
& Indian & 42 & 12.0 \\
Age & Others & 28 & 8.0 \\
& $19-21$ & 283 & 80.9 \\
& $22-24$ & 67 & 19.1 \\
& 25 and above & - & - \\
Year of Study & $1^{\text {st }}$ Year & 290 & 82.9 \\
& $2^{\text {nd }}$ Year & 42 & 12.0 \\
& $3^{\text {rd }}$ Year & 18 & 5.1 \\
& $1-3$ & 224 & 64.0 \\
& $4-6$ & 126 & 36.0 \\
Years of Using Facebook & $7-9$ & - & - \\
& 10 and above & - & - \\
& & &
\end{tabular}


Scale purification process is of paramount importance for assessing the reliability and validity of the measurement scales. This process involves evidence of theoretical content as well as validated empirical tests in order to evaluate the quality of the data. To this point, item analysis was carried out to ensure non-violation of normality assumptions, reliable and valid prior to multivariate analysis.

\subsection{Factor Analysis}

24 items were used to measure perceptions toward advertising on Facebook. Initial results of the factor analysis on the 24 items came out with four factors. Since items with loading of 0.45 and above and not cross loaded in other factors were accepted, one items from privacy were eliminated from the subsequent analyses. The remaining 23 items were re-analyzed and the result is presented in Table 2. The SPSS output is presented in Appendix. From the table, the KMO measure sampling adequacy values for the items was 0.666 .

Table 2. Factor analysis on perceptions towards advertising on Facebook

$\begin{array}{llllll}\text { ITEMS } & 1 & 2 & 3 & 4\end{array}$

\section{Perceived Interactivity}

- Advertisements in Facebook catch my attention among other content.

- I will visit a product website after being exposed to an ad in Facebook. $\quad .810$

- I will purchase a product advertised in Facebook.

- In my daily activities, I recognize brands as the ones that are advertised in Facebook.

- It is easier for me to remember brands advertised through other media channels such as TV and radio as opposed to the ones advertised in Facebook.

- Facebook advertising is a valuable source of information about latest fashion.

\section{Advertising Avoidance}

- I visit Facebook with a bit of plan of what I am going to do.

- I can list the advertisements that I can remember seeing on Facebook?

- Advertisement on Facebook tells me what persons like me are buying or using.

.566

- I felt that I was at risk of getting a virus by clicking onto a link in Facebook advertising.

- The position of advertisement is the biggest factor that prevents me from looking at ads on Facebook.

- I am the fan of at least one company or brand in Facebook advertising.

Credibility

- Advertising on Facebook provide accurate information about products/services.

- Facebook advertising insults the intelligence of the average consumer.

- Facebook advertising tells me which brands have the features I am looking for.

- There are too many exaggerations in Facebook advertising.

- Facebook should be used for promoting commercial products/ services.

- Because of Facebook advertising, people buy a lot of things that they do not really need.

\section{Privacy}

- Facebook advertising distorts the values of our youth. 
- Advertisements found on Facebook are tailored to your interests.

- Facebook advertising keeps me up to date about products/services $\quad 379$ available in the marketplaces.

- Advertisements on Facebook are intrusive.

- Users of Facebook will be forced to see the advertisement every time they logged in.

Eigenvalues

Percentages variance explained (\%)

Total variance explained (\%)

KMO

Bartlett's Test of Sphericity
.588

$\begin{array}{lll}5.836 & 3.780 & 2.400\end{array}$

1.879

25.37

16.44

10.43

8.17

60.41

.666

6444.109

3 items were used to measure perceptions toward Advertising on Facebook. Initial results of the factor analysis on the 3 items came out with one factor. Since items with loading of 0.45 and above and not cross loaded in other factors were accepted. The 3 items were re-analyzed and the result is presented in Table 3.

Table 3. Factor analysis on attitudes towards advertising on Facebook

\begin{tabular}{lc}
\multicolumn{1}{c}{ ITEMS } & Factor 1 \\
\hline - Given the scale, how would you rate your attitude toward advertising on & .685 \\
Facebook? & .667 \\
- Given the scale, how would you rate your attitude toward advertising on & \\
Facebook? & .712 \\
- Given the scale, how would you rate your attitude toward advertising on & $\mathbf{5 . 3 4 5}$ \\
Facebook? & $\mathbf{1 0 0}$ \\
Eigenvalues & \\
Percentages variance explained (\%) & $\mathbf{0 . 7 0 7}$ \\
Total variance explained (\%) & $\mathbf{1 1 7 5 . 5 9 0}$ \\
KMO & \\
Bartlett's Test of Sphericity &
\end{tabular}

\subsection{Reliability Analysis}

Acceptable ranges of reliability of most instruments are ranged from 0.7 to 0.9 . The closer the alphas to 1 , the better the instruments are. Sekaran (2000) suggested that the minimum acceptable reliability is at 0.60 . The Cronbach Alphas were computed for reliability testing. All the items for each construct of the study possess Cronbach $\alpha$ value above 0.60 except for one item in the dimension of privacy which possessed below 0.60 . Based on Nunally (1967), the items for each construct in the questionnaire are reliable and have internal consistency. The results for the reliability tests for this study are highly reliable as shown in Table 4 . 
Table 4. Results of reliability test

\begin{tabular}{ccc}
\hline VARIABLES & NUMBER OF ITEMS & CRONBACH'S ALPHA \\
\hline INDEPENDENT VARIABLES & & \\
Perceptions toward Advertising on Facebook & 23 & 0.770 \\
Perceived Interactivity & 6 & 0.781 \\
Advertising Avoidance & 6 & 0.643 \\
Credibility & 6 & 0.630 \\
Privacy & 5 & 0.544 \\
DEPENDENT VARIABLE & & 0.620 \\
\hline
\end{tabular}

\subsection{Hypothesis Results}

By using regression analysis, the following results were obtained (refer Table 5):

a) There is a significant positive relationship between perceived interactivity and attitude towards the advertisement on Facebook.

b) There is significant positive relationship between advertising avoidance and attitude towards the advertisement on Facebook.

c) There is significant positive relationship between advertising avoidance and attitude towards the advertisement on Facebook.

d) There is positive relationship between credibility and attitude towards the advertisement on Facebook.

Summary of the hypothesis results is presented in Table 6 .

Table 5. Multiple regression analysis

\begin{tabular}{ccccccc}
\hline & Model & \multicolumn{2}{c}{ Unstandardized Coefficients } & $\begin{array}{c}\text { Standardized } \\
\text { Coefficients }\end{array}$ & \multirow{2}{*}{ t } & Sig. \\
\cline { 3 - 5 } & & $\mathbf{B}$ & Std. Error & Beta & & \\
\hline $\mathbf{1}$ & (Constant) & 1.554 & .161 & & 9.635 & .000 \\
& Total Interactivity & .134 & .037 & .201 & 3.623 & .000 \\
& Total Privacy & .338 & .052 & .406 & 6.523 & .000 \\
& Total Avoidance & .368 & .047 & .389 & 7.873 & .000 \\
& Total Credibility & .001 & .045 & .001 & .020 & .232
\end{tabular}

a. Dependent Variable: Total Attitude

\begin{tabular}{llcc} 
Model & & \multicolumn{2}{c}{$\begin{array}{c}\text { Unstandardized } \\
\text { Coefficients }\end{array}$} \\
& & B & Std. Error \\
1 & (Constant) & 1.649 & .164 \\
& Total Perceptions & .350 & .051
\end{tabular}

$\begin{array}{ccc}\begin{array}{c}\text { Standardized } \\ \text { Coefficients } \\ \text { Beta }\end{array} & \mathbf{t} & \text { Sig. } \\ & 10.031 & .000 \\ .343 & 6.806 & .000\end{array}$

a. Dependent Variable: TotalAttitude 
Table 6. Summary of hypothesis results

\begin{tabular}{ccc}
\hline Regression & Result & Remark \\
\hline Perceived Interactivity & 0.000 & Supported \\
Advertising Avoidance & 0.000 & Supported \\
Credibility & 0.232 & Not Supported (Not significant) \\
Privacy & 0.000 & Supported \\
\hline
\end{tabular}

\subsection{Discussions}

Multiple regression results had partially supported the hypothesis i.e. there is relationship between perceptions toward advertising on Facebook and attitude towards the advertisement on Facebook. As expected, the perceived interactivity, privacy and advertising avoidance (three dimensions of perceptions toward advertising on Facebook) was found to be positively correlated with the dimension of attitude towards the advertisement on Facebook. Surprisingly, credibility (another dimension of perceptions toward advertising on Facebook) was not a significant predictor to attitude towards the advertisement on Facebook. It is essential to understand how Internet users perceive advertising on Facebook and what factors would affect their attitudes towards Internet advertising. Moreover, knowing what kind of content shall be put in the advertisement or in what way of advertisements shall be used would be helpful in enhancing marketing campaign plan.

When consumers are skeptical of the advertising messages they are receiving, or are skeptical of the media source of the message, they will not be motivated to process the information they are receiving. This may lead to coping responses from consumers such as gathering information from other sources or avoidance of the advertising message altogether (Obermiller et al., 2005). Unsuitable targeting techniques used by marketers in delivering messages may also lead to lack of trust. Johnson and Kaye (1998) stated that if consumers do not trust or believe the media then they are less likely to pay attention to it. Of late, there are a few reports that raised the same detrimental issue. An independent research, Webtrends (2011) reported that Facebook advertising performance based on CTR was very low ( 0.051 percent), although this may not affect the users but it definitely has a major impact on Facebook as a credible platform of advertising in the eyes of marketers. An online portal Readwrite Social also raised the issue of Facebook's credibility on the ground of its email scanning practice, albeit clarification by Facebook which pointing out an entirely different issue of privacy (Copeland, 2012).

Therefore, it is of paramount importance to ensure that resources should be more accurately allocated to the media or channel and sources must be clearly verified after knowing that users have concern about the credibility issue pertaining to some advertising media especially interactive ones like Facebook. This is because informational interactivity plays major role in web and online social network advertising since it is designed in a way that allows for a user to search for and locate information (Barnes, 2006).

\section{Limitations and Future Research}

As mentioned earlier, researchers who used limited-parent population could only justify the dimensions explaining the research framework without generalizing the findings and drawing conclusions about the overall public attitude towards advertising (Shavitt et al. 1998). In this particular study, any conclusion drawn from this limited-parent population sample (undergraduate students) would be misleading because the sample basis does not represent the total population despite the justifications as mentioned above. The findings, however, represent student perceptions from a sufficiently diverse range of faculties and subject areas where Facebook has been used. Thus, these exploratory findings represent a broad view of the issues that arise. Future research may consider using a more general and representative population of Internet users (i.e. raising the sample size).

\section{Conclusion and Implication}

Hirschman and Thompson (1997) opined that issues in vast areas of advertising must be tackled with a deeper understanding of consumers' relationships with advertising and the media. This present study fully supports this notion and, in addition, has discovered that any generic issues, such as what were uncovered in this study, must be seen from a specific media frame of reference (i.e. Facebook). In this study, consumers' perceptions such as perceived interactivity, privacy and advertising avoidance have great effects on the attitudes towards the advertising in Facebook. From the findings of this research, the marketers and advertising designers can also understand and pay more attention on how the users of Facebook perceive the advertisement when making advertising decisions. 
Besides providing literature for future scholarly research avenues, it is also hoped that this will be a good starting point for researchers to further examine attitudes towards other specific popular and hybrid advertising techniques like advergames and interactive digital television before jumping on the bandwagon.

\section{References}

Andrew, J. C., Lyonski, S., \& Durvasula, S. (1991). Understanding Cross-Cultural Student Perceptions of Advertising in General: Implications for Advertising Educators and Practitioners. Journal of Advertising, 20(2), 15-28.

Barnes, S. B. (2006). A privacy Paradox: Social Networking in the United States. First Monday, 11(9).

Bond et al. (2010). Social Media Advertising: An Investigation of Consumer Perceptions, Attitudes and Preference of Engagement. ANZMAC, 1-9.

Bonneau, \& Preibusch. (2009). The Privacy Jungle: On the Market for data Protection in Social Networks. In the eight Workshop on the Economics on Information Security (WEIS 2009).

Boyd, D. M., \& Ellison, N. B. (2007). Social Network Sites: Definition, History and Scholarship. Journal of Computer-Mediated Communication, 31(1), 210-230. http://dx.doi.org/10.1111/j.1083-6101.2007.00393.x

Calisir, F. (2003). Web Advertising vs. Other Media: Young Consumers' View. Internet Research: Electronic Networking Applications and Policy, 13(5), 356-363. http://dx.doi.org/10.1108/10662240310501630

Campbell, \& Wright, R. T. (2008). Shut-up I Don't Care: Understanding the Role of Relevance and Interactivity on Consumer Attitudes toward Repetitive Online Advertising. University of San Francisco.

Cho, C., \& Cheon, H. J. (2004). Why Do People Avoid Advertising on the Internet? Journal of Advertising, 33(4), 89-97.

Copeland, D. (2012). Facebook's Email Scanning isn't a privacy issue, it's a credibility issue. Retrieved December 31, 2012, from http://readwrite.com/2012/10/05/facebooks-email-scanning-isnt-a-privacy-issue-its-a-credibility-issue

Cranor et al. (2000). Beyond Concern: Understanding Net Users' Attitudes about Online Privacy. In I. Vogelsang, \& B. M. Compaine (Eds.), The Internet Upheaval: Rainsing Questions, Seeking Answers in Communications Policy (pp. 47-70). Cambridge, MA: MIT Press.

Gorard, S. (2001). Quantitative Methods in Educational Research: The Role of Numbers Made Easy. London: Continuum.

Gritten. A. (2007). Forum-Media Proliferation and Demands for New Forms of Research. International Journal of Market Research, 49(1), 15-23.

Ha, L., \& James, E. L. (1998). Interactivity Reexamined: A Baseline Analysis of Early Business Websites. Journal of Broadcasting and Electronic Media, 42(4), 457-474. http://dx.doi.org/10.1080/08838159809364462

Hadija, Z. (2008). Perceptions of Advertising in Online Social Networks: In Depth Interviews. The Rochester Institute of Technology, Department of Communication, College of Liberal Arts.

Hirschman, E. C., \& Thompson, C. J. (1997). Why Media Matter: Toward a Richer Understanding of Consumer' Relationships with Advertising and Mass Media. Journal of Advertising, 26(1), 43-60.

Johnson, T., \& Kaye, B. (1998). Cruising is Believing? Comparing Internet and Traditional News Sources on Media Credbility Measures. Journalism and Mass Communication Quarterly, 75(2), 325-340. http://dx.doi.org/10.1177/107769909807500208

Johnson, T., \& Kaye, B. (2002). Webelievabiliti: A Path Model Examining How Convenience and Reliance Predict Online Credibility. Journalism and Mass Communication Quarterly, 79(3), 619-642. http://dx.doi.org/10.1177/107769900207900306

Kelly, L. (2008). Teenagers' perceptions of advertising in the online social networking environment: An exploratory study. Masters by Research thesis, Queensland University of Technology. Retrieved from http://eprints.qut.edu.au/26966/

Lenhart et al. (2010). Social Media \& Mobile Internet Use among Teens and Young Adults. Pew Internet \& American Life Project.

Mangold, W. G., \& Faulds, D. J. (2009). Social Media: The New Hybrid Element of the Promotion Mix. 
Business Horizons, 5(4), 357-365. http://dx.doi.org/10.1016/j.bushor.2009.03.002

McDonald et al. (2009). A Comparative Study of Online Privacy Policies and Formats. In Proceedings of Privacy Enhancing Technologies (pp. 37-55). http://dx.doi.org/10.1007/978-3-642-03168-7_3

McDonald, A. M., \& Cranor, L. F. (2009). The Cost of Reading Privacy Policies. ISJLP, 4, 543-897.

Moore, J. J., \& Rodgers, L. R. (2005). An Examination of Advertising Credibility and Skepticism in Five Different Media Using the Persuasion Knowledge Model, American Academy of Advertising Conference Proceedings, Januari 1, 10.

Nunally, J. C. (1967). Psychometric Theory. New York: MCGraw Hill.

Obermiller et al. (2005). Ad Skepticism: The Consequences of Disbelief. Journal of Advertising, 34(3), 7-17.

Ragan, S. (2009). Privacy Issues Plague Facebook Users-Yet Again. Retrieved November 16, 2009, from http://www.thetechherald.com/article.php/200938/4431/Privacy-issues-plague-Facebook-users- $\%$ E2\%80\%9 $3 \%$-yet-again

Roberts (2010). Privacy and Perceptions: How Facebook Advertising Affects its Users. The Elon Journal of Undergraduate Research in Communications, 1(1).

Sekaran, U. (2000). Applied Business Research: A Skill Buildnig Approach. John Wiley \& Sons, USA.

Shavitt, S., Lowrey, P., \& Haefner, J. (1998). Public Attitudes Toward Advertising: More Favourable Than You Might Think. Journal of Advertising Research, (Jul/Aug), 7-22.

Smith et al. (2010). The ECAR Study of Undergraduate Students and Information Technology, (research Study, Vol. 6). Boulder, CO: EDUCAUSE center for Applied Research.

Speck, P. S., \& Elliott, M. T. (1997). Predictors of Advertising Avoidance in Print and Broadcast Media. Journal of Advertising, 26(3), 61-76.

Webtrends. (2011). Facebook's Advertisign Performance Benchmark \& Insights, WebTrends. Retrieved 30 $\begin{array}{lll}\text { December, } & 2012, & \text { from }\end{array}$ https://docs.google.com/viewer?url=http $\% 3 \mathrm{~A} \% 2 \mathrm{~F} \% 2 \mathrm{Ff} . \mathrm{cl} .1 \mathrm{\%} \% 2 \mathrm{Fitems} \% 2 \mathrm{~F} 2 \mathrm{~m} 1 \mathrm{y} 0 \mathrm{~K} 2 \mathrm{~A} 062 \mathrm{x} 0 \mathrm{e} 2 \mathrm{k} 442 \mathrm{l} \% 2 \mathrm{Ff}$ acebook-advertising-performance.pdf

Yaakop, A., \& Hemsley-Brown, J. (2011). Attitudes towards Advertising: Does Traditional Media still have its Place in the Future? 1st International Conference on Accounting, Business and Economics (ICABEC2011) Proceedings, October 2011.

Yang, C. C. (2000). Taiwanese Students' Attitudes Towards and Belief About Advertising. Journal of Marketing Communications, 6, 171-183. http://dx.doi.org/10.1080/13527260050118667

Zeng et al. (2009). Social Factors in User Perceptions and Responses to Advertising in Online Social Networking Communities. Journal of Interactive Advertising, 10(1), 1-13. 